\title{
Dual-pattern communication system using DBR laser in the intensity-modulation and direct-detection system
}

\author{
Borui Xu๑ ${ }^{\text {a,b }}$ Jiazheng Sun, ${ }^{\text {a,b }}$ Huashun Wen, ${ }^{\text {a }}$ and Ninghua $\mathrm{Zhu}^{\mathrm{a}}{ }^{*}$ \\ ${ }^{a}$ Institute of Semiconductors Chinese Academy of Sciences, State Key Laboratory \\ on Integrated Optoelectronics, Beijing, China \\ ${ }^{b}$ University of Chinese Academy of Sciences, College of Materials Science \\ and Opto-Electronic Technology, Beijing, China
}

\begin{abstract}
We propose a flexible dual-pattern communication system based on the existing intensity modulation and direct detection system, which exploits the wavelength tuning characteristic of the distributed Bragg reflector (DBR) laser. At the transmitter side of the system, the DBR laser is wavelength tuned by the wavelength coding signal and intensity modulated by the radio frequency (RF) signal. At the receiver side, the RF signal is detected directly by photodetector 1 , while the wavelength coding signal is recovered by a specific optical filter. The proposed system is theoretically analyzed and verified at the $\mathrm{C}$ band experimentally. We successfully obtained clear eye openings for the 10- and $25-\mathrm{Gb} / \mathrm{s}$ nonreturn-to zero signal. The waveform graphs of the 10- and $100-\mathrm{kHz}$ wavelength coding signal were also measured. The two transmission patterns did not interfere with each other no matter in the back-to-back or $5 \mathrm{~km}$ case. () The Authors. Published by SPIE under a Creative Commons Attribution 4.0 International License. Distribution or reproduction of this work in whole or in part requires full attribution of the original publication, including its DOI. [DOI: 10.1117/1.OE.60.11.116103]
\end{abstract}

Keywords: dual-pattern communication system; intensity modulation and direct detection; wavelength coding signal; DBR laser.

Paper 20210875 received Aug. 9, 2021; accepted for publication Oct. 21, 2021; published online Nov. 10, 2021.

\section{Introduction}

The arrival of the information age has accelerated the growth of Internet data traffic, and the optical communication has been playing an increasingly important role in the long, metro, and short transmission networks. At the same time, the secure transmission of optical network systems has attracted widespread interests. ${ }^{1}$ Physical-layer encryption using optical approaches is a safe optical communication method in which the signal is protected at the physical layer to prevent attackers from discovering the data transmission. ${ }^{2,3}$ The mainly physical-layer encryption methods for optical communication are chaos communication, optical code-division multiplexing, and hidden communication. ${ }^{4-11}$ Optical hidden communication hides the secure signal in public signal and transmits them in a common channel. It is difficult for attackers to perceive the existence of the secure signal. In addition, the optical hidden communication can use the existing public optical network to realize the transmission of secure signal. Compared with other methods for secure communication, the hidden communication is more attractive due to its low cost, mature technical conditions, and flexible implementation advantages. ${ }^{12,13}$ On the other hand, intensity modulation and direct detection (IM-DD) systems are widely used due to their low power consumption and low cost, ${ }^{14}$ and the distributed Bragg reflector (DBR) laser is promising owing to its easy construction, compact size, and good long-term reliability. ${ }^{15-17}$

In this paper, we proposed a dual-pattern communication system based on the IM-DD system using the DBR laser to establish a high-speed, low cost, and secure optical communication system. The dual-pattern communication system transmits the radio frequency (RF) signal and the wavelength coding signal simultaneously, and the wavelength coding signal can be hidden in the RF signal. The dual-pattern communication system can be used for the hidden communication, when the wavelength coding signal acts as the secure signal and the RF signal acts as the public signal.

*Address all correspondence to Ninghua Zhu, nhzhu@semi.ac.cn 
At the transmitter side of the dual-pattern communication system, the DBR laser is wavelength modulated by the wavelength coding signal while transmitting the RF signal by an external Mach-Zehnder modulator (MZM). At the receiver side, the RF signal is detected by a standard IM-DD scheme, and the wavelength coding signal is detected by an optical filter and a photodetector (PD). The optical filter is used as an optical discriminator. We also carried out an experiment to study the transmission performance of the dual-pattern communication system. To simplify the system, we chose a square wave signal as the wavelength coding signal. The eye diagrams of the nonreturn to zero (NRZ) signal and the waveform graphs of the wavelength coding signal were measured to evaluate the performance of the system. The experiment results show that the IM-DD communication and the wavelength coding communication can be carried out at the same time without interfering with each other. Therefore, when the wavelength coding signal is hidden in IM-DD communication as the secure signal, the wavelength coding signal will not be found unless utilizing a specific optical filter.

\section{Device Characterization and Theory of Dual-Pattern Communication}

The light source we used is a three-section DBR laser with a $1300-\mu \mathrm{m}$ cavity length including gain section, grating section, and phase section. These three sections have different functions. The gain section mainly controls the output power whereas the other two sections mainly control the lasing wavelength. The wavelength tuning speed of the DBR laser is $10 \mathrm{~ms}$.

The light-current characteristic of the commercial tunable DBR laser at $25^{\circ} \mathrm{C}$ is shown in Fig. 1(a). The threshold current of the DBR laser is $40 \mathrm{~mA}$, and the value of slope efficiency is $0.2 \mathrm{~W} / \mathrm{A}$ when the grating current $I_{\text {grating }}$ and phase current $I_{\text {phase }}$ are both fixed at $0 \mathrm{~mA}$. When the $I_{\text {grating }}$ and $I_{\text {phase }}$ are biased at 30 and $2.4 \mathrm{~mA}$, respectively, the threshold current of the DBR laser is $52 \mathrm{~mA}$, and the slope efficiency is $0.18 \mathrm{~W} / \mathrm{A}$. Figure 1(b) shows the optical spectra of the DBR laser with the $I_{\text {grating }}$ and $I_{\text {phase }}$ fixed at 30 and $2.4 \mathrm{~mA}$. Figure 1(b) shows that the wavelength of the laser moves to longer wavelengths as the gain current increases. The wavelength redshift caused by the injection current in gain section is the result of the thermal effect. Figure 1(c) shows the output power of the laser versus $I_{\text {grating }}$ when the gain current $I_{\text {gain }}$ is $100 \mathrm{~mA}$ and $I_{\text {phase }}$ is $2.4 \mathrm{~mA}$. Obviously, the output power is inversely proportional to the grating current. This is because the current injected into the grating section increases the loss of the laser. The lasing wavelength and the corresponding side mode suppression ratio (SMSR) of the DBR laser are shown in Fig. 1(d). The wavelength is tuned from 1552.81 to $1544.72 \mathrm{~nm}$ with $I_{\text {grating }}$ increasing from 0 to $70 \mathrm{~mA}$, and the SMSR is $>35 \mathrm{~dB}$. The wavelength blueshift is the result of the free carrier plasma effect, which leads to a decrease of the effective refractive index and a blueshift of the grating reflection spectrum. At the same time, the injection of the grating current will change the power of the main mode and the reflectivities of the main mode and side mode, which will cause the SMSR to change. ${ }^{18}$

The tuning characteristic of the phase section is to achieve quasicontinuous wavelength tuning without changing the output power. ${ }^{19}$ Figure 1 (e) shows the output power of the laser versus $I_{\text {phase }}$. Figure 1(f) shows the lasing wavelength and the SMSR as a function of $I_{\text {phase }}$ with $I_{\text {gain }}$ and $I_{\text {grating }}$ biased at 100 and $30 \mathrm{~mA}$. When the side mode and the main mode experience equal losses, the side mode will take over and become the new main mode and the SMSR is at its worst. It can be seen from Figs. 1(e) and 1(f) that when $I_{\text {phase }}$ increases from 1 to $3.7 \mathrm{~mA}$, the wavelength decreases continuously, and the corresponding output power of the DBR laser just fluctuates in a small range. This is because that the phase section realizes wavelength tuning by changing the cavity mode.

As shown in Fig. 2(a), based on the quasicontinuous tuning characteristics obtained in Figs. 1(e) and 1(f), a specific lasing wavelength corresponds to a specific phase current. If we add a M-ary pulse amplitude modulation (M-PAM) wavelength coding signal to the phase section of the DBR, the lasing wavelength will be tuned among $\lambda_{1}, \lambda_{2}, \ldots, \lambda_{m}$. The angular frequency of the light wave can be expressed as the convolution of the sum of $m$ rectangle functions and a comb function: ${ }^{20}$ 
Xu et al.: Dual-pattern communication system using DBR laser in the intensity-modulation...
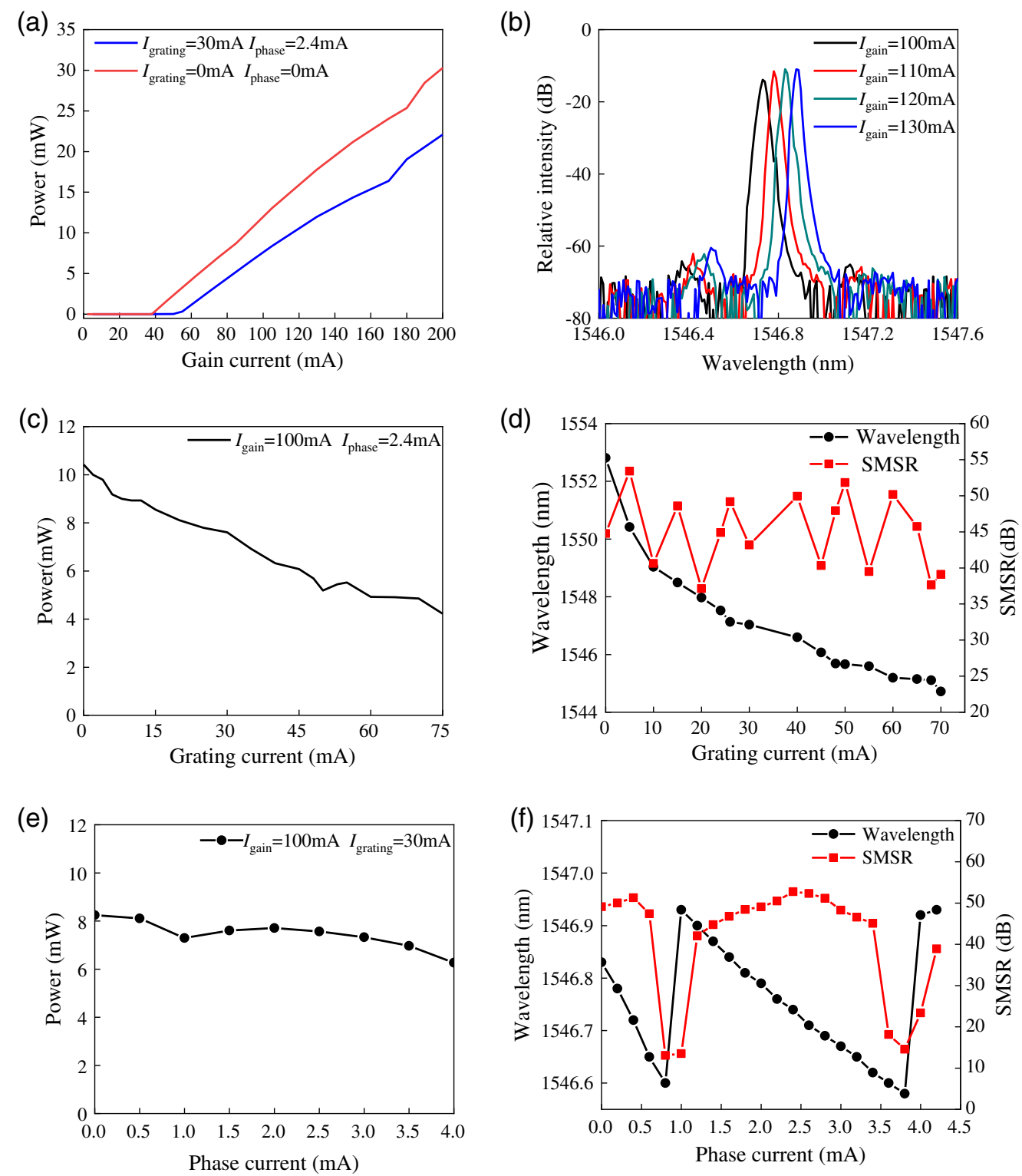

Fig. 1 (a) The light output power as a function of the gain current, (b) typical optical spectra, (c) the light output power as a function of the grating current, (d) wavelength tuning properties of the grating section, (e) the light output power as a function of the phase current, and (f) wavelength tuning properties of the phase section.

$$
\begin{aligned}
\omega(t)= & {\left[\omega_{1} \operatorname{rect}\left(\frac{t-t_{0} / 2}{t_{0}}\right)+\omega_{2} \operatorname{rect}\left(\frac{t-3 t_{0} / 2}{t_{0}}\right)+\cdots+\omega_{m} \operatorname{rect}\left(\frac{t-(2 m-1) \cdot t_{0} / 2}{t_{0}}\right)\right] } \\
& * \operatorname{comb}\left(\frac{t}{m t_{0}}\right),
\end{aligned}
$$

where the $\omega_{1}, \omega_{2}, \ldots, \omega_{m}$ are the angular frequencies of the light wave corresponding to wavelengths $\lambda_{1}, \lambda_{2}, \ldots, \lambda_{m}$. Considering the wavelength tuning characteristic of phase section, the electric field of the light wave emitted by the DBR laser is written as

$$
E(t)=E_{0} \exp \left[j\left(\int_{0}^{t} \omega(\tau) \mathrm{d} \tau+\varphi(t)\right)\right]
$$


where $E_{0}$ is the amplitude of the electric field and $\varphi(t)$ describes the laser phase noise. Then, the light wave is external modulated by the MZM. The optical field at the output of MZM is ${ }^{21}$

$$
E_{\text {out }}(t)=\frac{1}{2} E(t) \cdot\left\{\exp \left[j\left(\beta \cos \left(\omega_{\mathrm{RF}} t\right)+\frac{\psi}{2}\right)\right]+\exp \left[-j\left(\beta \cos \left(\omega_{\mathrm{RF}} t\right)+\frac{\psi}{2}\right)\right]\right\}
$$

where $\beta$ is the RF modulation index of the MZM, $\omega_{\mathrm{RF}}$ is the angular frequency of the RF signal, and $\Psi$ is the phase difference between the two arms of the MZM. The output current of the PD is proportional to optical power: ${ }^{22,23}$

$$
I_{\mathrm{PD}}=R \cdot P,
$$

where $R$ is the responsivity of PD. At the receiver side, photodetector 1 (PD1) is used to directly detect the optical signal, and the optical current $I_{\mathrm{PD} 1}$ generated in the PD1 can be written as

$$
I_{\mathrm{PD} 1} \propto \frac{1}{2} E_{0}^{2}-E_{0}^{2} \sin (\varphi) J_{1}(2 \beta) \cos \left(\omega_{\mathrm{RF}} t\right)
$$

So, the RF signal can be detected directly by the PD1. An optical filter is set before PD2 as an optical discriminator to recover the M-PAM wavelength coding signal. As shown in Fig. 2(b), when the lasing wavelength located on the falling edge of the optical filter, the optical power after passing through the optical filter will decrease as the wavelength increases. Therefore, the optical current $I_{\mathrm{PD} 2}$ generated in the PD2 is written as

$$
I_{\mathrm{PD} 2} \propto E_{0}^{2}(a-b \lambda) .
$$

The use of optical filter makes the output current of the PD2 determined by the wavelength, that is, determined by the wavelength coding signal. So, the wavelength coding signal can be detected directly by the optical filter and PD2.

According to the aforementioned theory, we propose a dual-pattern communication system. The system transmits two communication patterns at the same time, namely the IM-DD communication and the wavelength coding communication. The IM-DD communication transmits the RF signal fed into the MZM. The wavelength coding communication transmits the wavelength coding signal fed into the phase section of the DBR. Since the phase section of the DBR achieves wavelength tuning by changing the cavity mode, modulating the phase current only changes the laser wavelength without changing the output power. ${ }^{19}$ Therefore, the transmission of the wavelength coding signal does not affect the communication quality of the IM-DD system. For further application, when the wavelength-coded signal is hidden in the IM-DD system, the dual-mode communication system can be used for hidden communication.
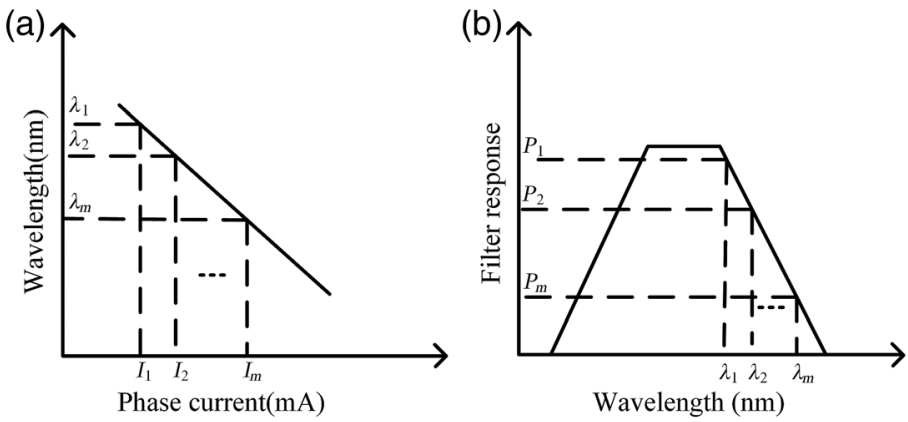

Fig. 2 Operating principle of the wavelength coding communication. (a) Wavelength tuning characteristic of the phase section. (b) The optical filter realizes the conversion from the wavelength tuning to the intensity modulation. 


\section{Experimental Results}

The schematic diagram of the dual-pattern communication system we proposed is shown in Fig. 3. The DBR laser is driven by the current source. The wavelength coding signal, generated by the wavelength coding signal source, is fed into the current source to modulate the phase current of DBR. Then, the light wave is sent to the MZM. The MZM is modulated by the $\mathrm{RF}$ signal, which is generated by the pulse pattern generator (PPG). After a transmission through the single mode fiber (SMF), a 10-dB optical coupler is used to split the optical signal into two branches. The $90 \%$ of the optical signal are sent to PD1 directly to detect the RF signal. The $10 \%$ of the optical signal enter PD2 after being processed by the optical filter. The filter realizes the conversion from wavelength tuning to intensity modulation. Therefore, the PD2 can recover the wavelength coding signal.

A proof-of-concept experiment was carried out to verify the proposed system. In the experiment, the $I_{\text {gain }}$ and $I_{\text {grating }}$ were biased at 100 and $30 \mathrm{~mA}$, respectively. The $I_{\text {phase }}$ was $1.1 \mathrm{~mA}$ and the output power measured at the pig-tail fiber was $7.3 \mathrm{~mW}$. The injection current of the phase section was modulated by the wavelength coding signal, which was generated by Agilent 33250A. For simplicity, we chose a square wave signal as the wavelength coding signal. The voltage levels of $V_{\text {high }}$ and $V_{\text {low }}$ were 25 and $0 \mathrm{mV}$, respectively. The corresponding $I_{\text {phase-high }}$ and $I_{\text {phase-low }}$ were 3.1 and $1.1 \mathrm{~mA}$, respectively. The working wavelengths of the DBR laser were $\lambda_{1} 1546.6 \mathrm{~nm}$ and $\lambda_{2} 1546.9 \mathrm{~nm}$. Figure 4(a) shows the optical spectrum of light waves with wavelengths $\lambda_{1}$ and $\lambda_{2}$. The wavelength-tuning light wave was externally modulated by the MZM. We set the pseudorandom bit sequence (PRBS) NRZ signal generated by PPG (Anristu MP1900A) as the RF signal. Then, the optical signal was transmitted to the receiver side through the SMF, and the dispersion factor of the SMF was $17 \mathrm{ps} / \mathrm{nm} \bullet \mathrm{km}$.

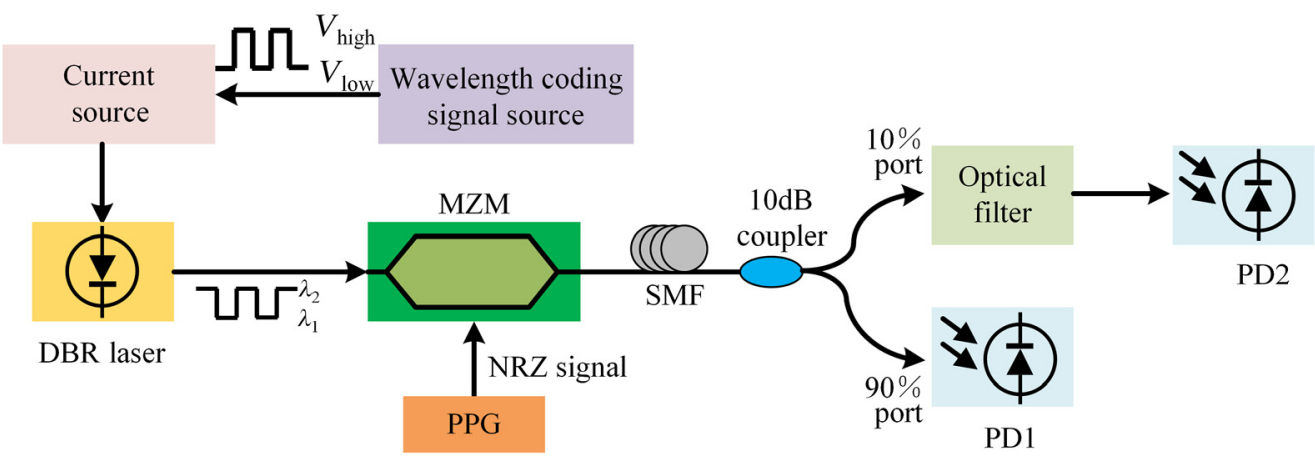

Fig. 3 Schematic of the dual-pattern communication system. PPG, pulse pattern generator; MZM, Mach-Zehnder modulator; SMF, single-mode fiber; PD, photodiode.

(a)

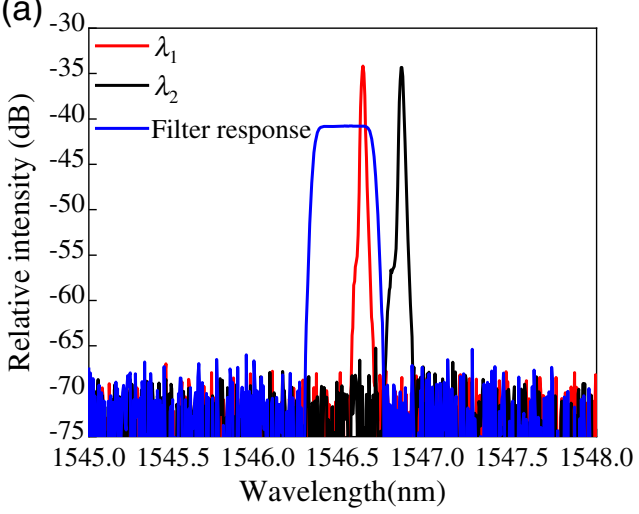

(b)

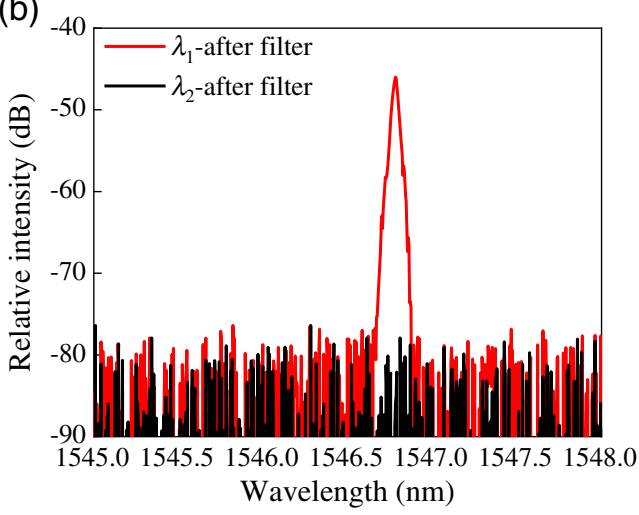

Fig. 4 (a) The optical spectra of $\lambda_{1}, \lambda_{2}$, and the measured optical filter response. (b) The optical spectra of $\lambda_{1}$ and $\lambda_{2}$ after passing through the optical filter. 
For the $90 \%$ branch, the optical signal was sent to PD1 directly to detect the PRBS NRZ signal. The bandwidth and sensitivity factor of the PD1 were $40 \mathrm{GHz}$ and $0.7 \mathrm{~A} / \mathrm{W}$, respectively. For the $10 \%$ branch, an optical bandpass filter was used to serve as an optical discriminator. The response of the optical filter is also shown in Fig. 4(a), which was measured by a broadband optical source (Lightcomm ASE) and an optical spectrum analyzer (Advantest Q8384). The optical spectrum of light waves with wavelengths $\lambda_{1}$ and $\lambda_{2}$ after passing through the optical filter is shown in Fig. 4(b). It can be seen from Fig. 4(b) that only the light wave with wavelength $\lambda_{1}$ passed through the filter, whereas the light wave with wavelength $\lambda_{2}$ was filtered out. When the wavelength was $\lambda_{1}$, the output current of the PD2 was high. When the wavelength was $\lambda_{2}$, the PD2 had no current output. Therefore, the square wave signal, namely the wavelength coding signal, was detected by the PD2.

The small-signal modulation response of IM-DD system corresponding to $\lambda_{1} 1546.6 \mathrm{~nm}$ and $\lambda_{2} 1546.9 \mathrm{~nm}$ was measured to explore the communication capacity of the IM-DD system. Figures $5(\mathrm{a})-5(\mathrm{~d})$ indicate that the frequency response of $\lambda_{1}$ is the same as $\lambda_{2}$ in the case of back-to-back (BtB), 1, 5, and $10 \mathrm{~km}$. It is verified that the communication quality of the IM-DD communication is not affected by the wavelength coding communication. Due to the power fading induced by the optical dispersion and double sidebands modulation, we observed the frequency dips of the frequency response in Figs. 5(c) and 5(d). The 3-dB bandwidth of the IM-DD system was $30,28,13$, and $8 \mathrm{GHz}$ in the case of $\mathrm{BtB}, 1,5$, and $10 \mathrm{~km}$, respectively. With the increasing of the fiber length, the IM-DD system suffered from severer power fading effect, thereby resulting in a smaller $3-\mathrm{dB}$ bandwidth. Considering that we will perform the transmission of $25 \mathrm{~Gb} / \mathrm{s} \mathrm{NRZ}$ signal, ${ }^{24}$ we choose the fiber length of $5 \mathrm{~km}$ in the following experiment.

The eye diagrams of the PRBS NRZ signal detected by PD1 were measured to evaluate the communication performance of the IM-DD system. Figure 6(a) shows the BtB eye diagrams of the PRBS NRZ signal at a speed of $10 \mathrm{~Gb} / \mathrm{s}$ when the wavelength coding signal source operates at different frequencies. It can be seen from Fig. 6(a) that all the eyes open clearly.
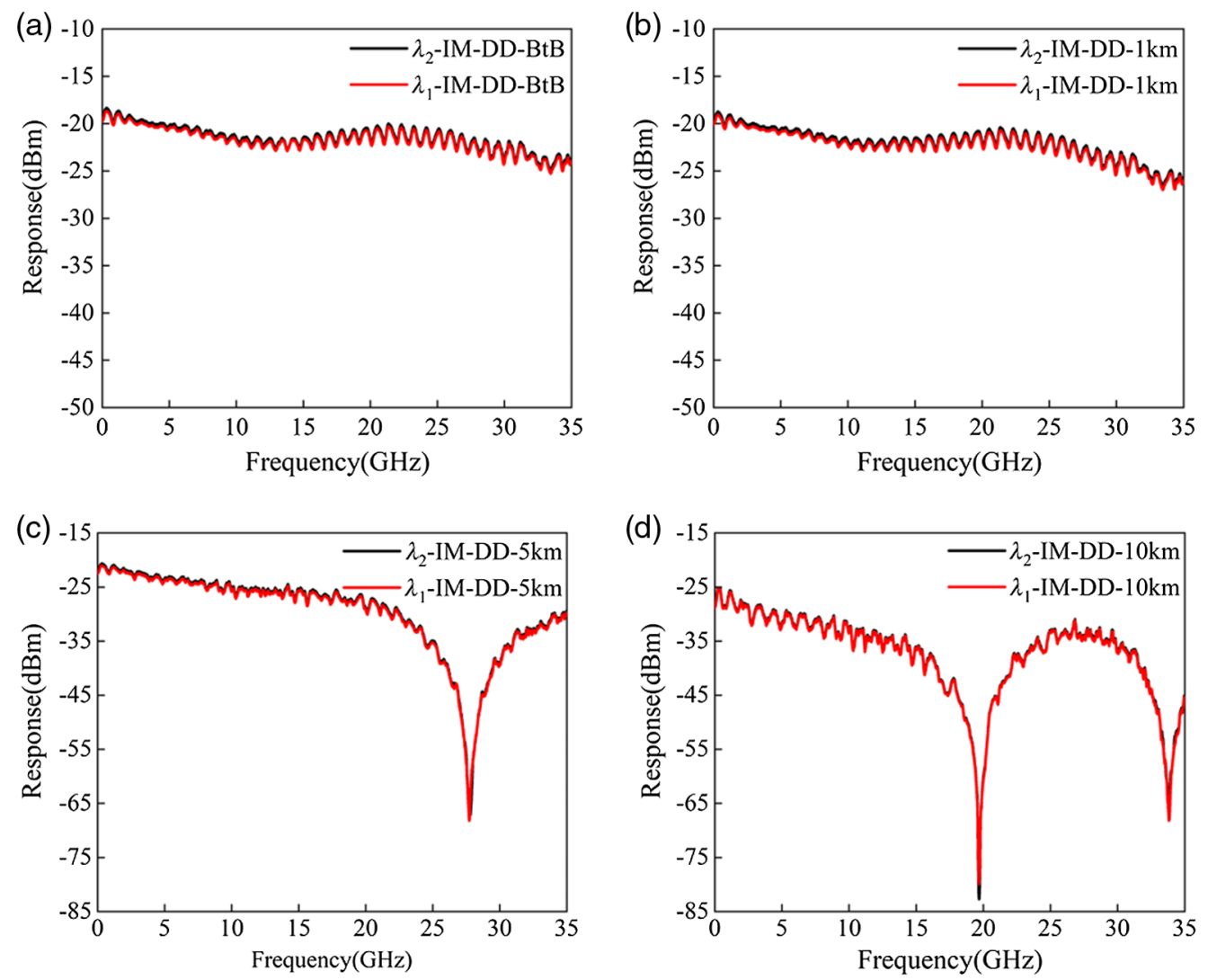

Fig. 5 The frequency response of the IM-DD system corresponding to $\lambda_{1} 1546.6 \mathrm{~nm}$ and $\lambda_{2} 1546.9 \mathrm{~nm}$ in the case of BtB, 1, 5, and $10 \mathrm{~km}$. 
(a) $1.1 \mathrm{~mA}-10 \mathrm{~Gb} / \mathrm{s}-\mathrm{BtB}$

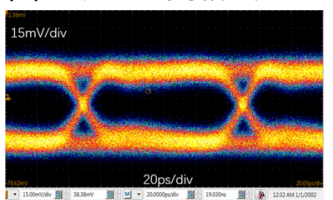

$10 \mathrm{kHz}-10 \mathrm{~Gb} / \mathrm{s}-\mathrm{BtB}$

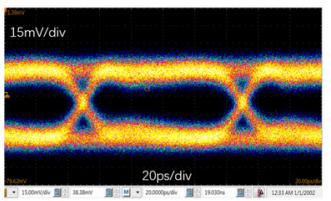

$100 \mathrm{kHz}-10 \mathrm{~Gb} / \mathrm{s}-\mathrm{BtB}$

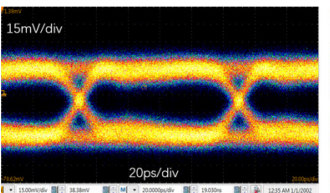

(b) $1.1 \mathrm{~mA}-25 \mathrm{~Gb} / \mathrm{s}-\mathrm{BtB}$

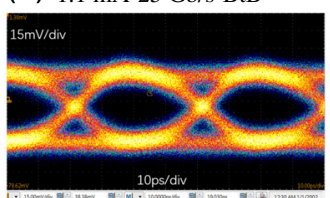

$10 \mathrm{kHz}-25 \mathrm{~Gb} / \mathrm{s}-\mathrm{BtB}$

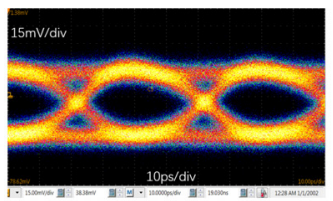

$100 \mathrm{kHz}-25 \mathrm{~Gb} / \mathrm{s}-\mathrm{BtB}$

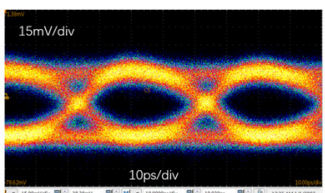

(c) $1.1 \mathrm{~mA}-10 \mathrm{~Gb} / \mathrm{s}-5 \mathrm{~km}$

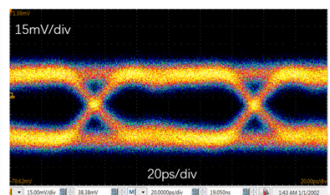

$10 \mathrm{kHz}-10 \mathrm{~Gb} / \mathrm{s}-5 \mathrm{~km}$

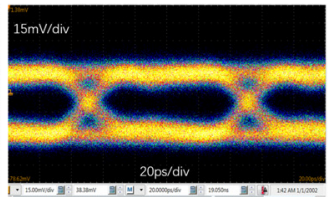

$100 \mathrm{kHz}-10 \mathrm{~Gb} / \mathrm{s}-5 \mathrm{~km}$

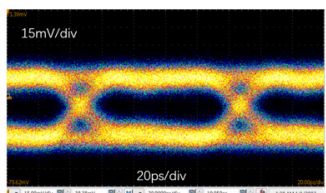

(d) $1.1 \mathrm{~mA}-25 \mathrm{~Gb} / \mathrm{s}-5 \mathrm{~km}$

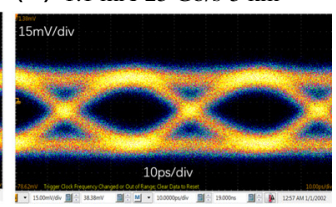

$10 \mathrm{kHz}-25 \mathrm{~Gb} / \mathrm{s}-5 \mathrm{~km}$

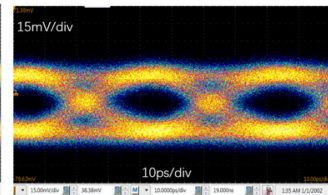

$100 \mathrm{kHz}-25 \mathrm{~Gb} / \mathrm{s}-5 \mathrm{~km}$

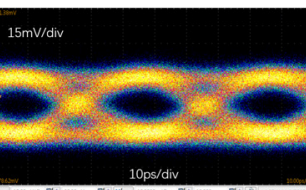

Fig. 6 The eye diagrams of the PRBS NRZ signal for (a) $10 \mathrm{~Gb} / \mathrm{s}$ BtB transmission, (b) $25 \mathrm{~Gb} / \mathrm{s}$ BtB transmission, (c) $10 \mathrm{~Gb} / \mathrm{s} 5-\mathrm{km}$ transmission, and (d) $25 \mathrm{~Gb} / \mathrm{s} 5-\mathrm{km}$ transmission.

The transmission of the wavelength coding signal did not deteriorate the NRZ signal. The measured 25-Gb/s BtB eye diagrams are shown in Fig. 6(b). There was also no evident difference between the system with wavelength coding signal and the system without wavelength coding signal for 25-Gb/s NRZ signal eyes. Therefore, the IM-DD communication will not be affected by the wavelength coding communication. Apart from the BtB situation, we also explored the transmission performance of the IM-DD communication in the case of $5 \mathrm{~km}$. Figure 6(c) shows the eye diagrams of $10-\mathrm{Gb} / \mathrm{s}$ NRZ signal after $5-\mathrm{km}$ transmission. All the eyes were open and clear. The eye diagrams corresponding to 10 and $100 \mathrm{kHz}$ wavelength coding signal were the same as the eye diagrams without wavelength coding signal. Figure 6(d) shows the eye diagrams of $25-\mathrm{Gb} / \mathrm{s}$ NRZ signal after $5-\mathrm{km}$ transmission. The extinction ratio of the eyes corresponding to 10 and $100 \mathrm{kHz}$ wavelength coding signal was slightly decreased due to the dispersion of the optical fiber.

The waveform graphs for the wavelength coding signal are exhibited in Figs. 7(a)-7(d) to evaluate the communication performance of the wavelength coding communication. The measured wavelength coding signal frequency was consistent with the square wave frequency. Figures 7(a)-7(d) show that the wavelength coding signals at $10 \mathrm{kHz}$ are standard square waves, whereas the square waves are distorted at $100 \mathrm{kHz}$. This is because that the wavelength tuning

(a) ${ }_{120}$

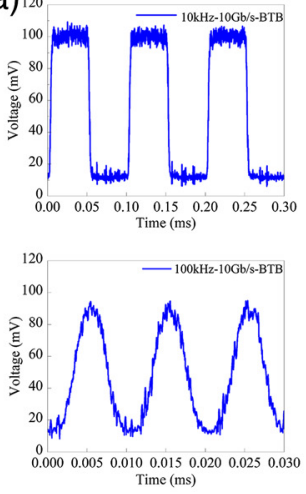

(b) ${ }_{120}$
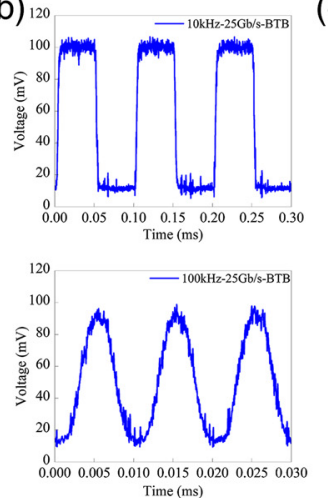

(c) ${ }_{120}$
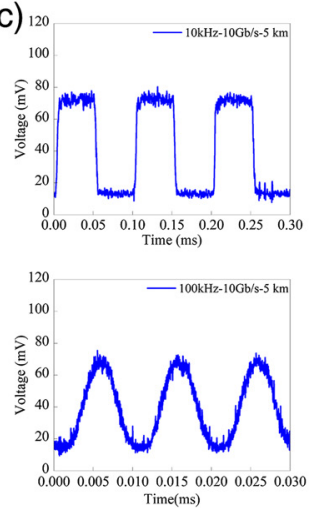

(d) ${ }_{120}$
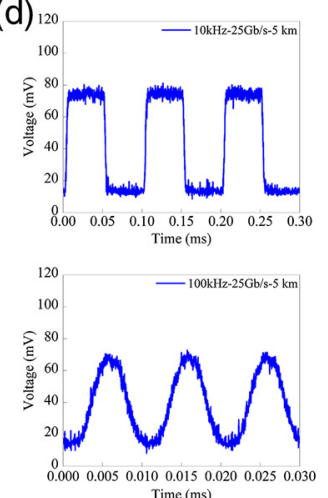
Time (ms)

Fig. 7 The waveform graphs of the signal detected by PD2 at 10 and $100 \mathrm{kHz}$ corresponding to (a) $10 \mathrm{~Gb} / \mathrm{s} \mathrm{NRZ} \mathrm{signal} \mathrm{BtB} \mathrm{transmission,} \mathrm{(c)} 25 \mathrm{~Gb} / \mathrm{s}$ NRZ signal BtB transmission, (b) $10 \mathrm{~Gb} / \mathrm{s}$ NRZ signal 5-km transmission, and (d) $25 \mathrm{~Gb} / \mathrm{s}$ NRZ signal 5-km transmission. 
speed of the DBR laser is $10 \mathrm{~ms}$, which limits the wavelength switching speed of $\lambda_{1}$ and $\lambda_{2}$. Using a DBR laser with a fast wavelength tuning speed can significantly increase the rate of wavelength coding signals. Shortening the cavity length, reducing the thermal effect, and adopting the pulse signal with pre-emphasis to tune the DBR laser can make the tuning rate of the DBR laser reach the ns level. ${ }^{25,26}$ Figure 7 (a) shows the 10 - and $100-\mathrm{kHz}$ wavelength coding signal detected by PD2 under the BtB transmission for 10-Gb/s NRZ signal. The 10- and 100$\mathrm{kHz}$ wavelength coding signal detected by PD2 under the BtB transmission for $10-\mathrm{Gb} / \mathrm{s} \mathrm{NRZ}$ signal is shown in Fig. 7(b). Comparing the wavelength coding signal corresponding to 10- and 25-Gb/s NRZ signals in Figs. 7(a) and 7(b), there is no difference in the wavelength coding signals when the IM-DD communication operates at different modulation speeds. It is verified that the IM-DD communication did not disturb the wavelength coding communication. Figures 7(c) and 7(d) show the wavelength coding signal detected by PD2 in the case of $5 \mathrm{~km}$ transmission. The waveform of the signal was similar to the case of BtB. However, the voltage of the signal decreased due to the transmission loss of the fiber.

\section{Conclusions and Discussions}

We proposed a dual-pattern communication system based on the IM-DD communication system. The dual-pattern communication system was realized by utilizing the wavelength tuning of the phase section of the DBR laser. On the other hand, the wavelength coding signal can be hidden in the IM-DD system to realize the hidden communication. We also verified the dual-pattern communication system experimentally. The eye diagrams of the PRBS NRZ signal and the waveform graphs of the wavelength coding signal were measured to evaluate the communication performance of the system. The system carried out the wavelength coding communication and the IMDD communication at the same time without interfering with each other. However, the optical dispersion and the wavelength tuning speed of the DBR laser are the main limitations of the dualpattern communication system. It is necessary to compensate the dispersion of the fiber and improve the wavelength tuning speed of the DBR laser in real-world applications. A fast wavelength tuning speed of the DBR can be achieved by shortening the cavity length, reducing the thermal effect, and adopting the pulse signal with pre-emphasis to tune the DBR laser.

\section{Acknowledgments}

This work was supported by the National Key Research and Development Program of China (2019YFB2203104, 2020YFB2205801, 2018YFB2201101), the National Natural Science Foundation of China (61805231, 61835010), Beijing Youth Fund (4214082), Chinese National Key Basic Research Special Fund (2018YFE0201200), the Strategic Priority Research Program of Chinese Academy of Sciences (XDB43000000), and Beijing Municipal Natural Science Foundation (4214082).

\section{References}

1. L. Yi et al., "Secure optical communication using stimulated Brillouin scattering in optical fiber," Opt. Commun. 290, 146-151 (2013).

2. T. Zhang et al., "High speed data security enabled by stimulated Brillouin scattering in optical fiber," in Asia Commun. and Photonics Conf. (2012).

3. L. L. Yi et al., "Power-penalty-free all-optical decryption using stimulated brillouin scattering in optical fiber," Laser Phys. Lett. 10(4), 045102 (2013).

4. F. Rogister et al., "Secure communication scheme using chaotic laser diodes subject to incoherent optical feedback and incoherent optical injection," Opt. Lett. 26(19), 1486-1488 (2001).

5. N. Li et al., "Chaotic optical cryptographic communication using a three-semiconductorlaser scheme," J. Opt. Soc. Am. B 29(1), 101-108 (2012).

6. M. Virte et al., "Deterministic polarization chaos from a laser diode," Nat. Photonics 7(1), 60-65 (2013). 
Xu et al.: Dual-pattern communication system using DBR laser in the intensity-modulation...

7. A. N. Pisarchik and F. R. Ruiz-Oliveras, "Optical chaotic communication using generalized and complete synchronization," IEEE J. Quantum Electron. 46(3), 279-284 (2010).

8. A. Argyris et al., "Chaos-based communications at high bit rates using commercial fibreoptic links," Nature 438(7066), 343-346 (2005).

9. M. Tomamichel et al., "Tight finite-key analysis for quantum cryptography," Nat. Commun. 3, 634 (2012).

10. B. B. Wu, P. R. Prucnal, and E. E. Narimanov, "Secure transmission over an existing public WDM lightwave network," IEEE Photonics Technol. Lett. 18(17), 1870-1872 (2006).

11. A. G. Sabbagh and M. M. Kakhki, "Performance analysis of two-level asynchronous optical CDMA systems utilizing wrapped OPPM," J. Lightwave Technol. 32(1), 122-129 (2014).

12. B. Wu and E. Narimanov, "Secure stealth transmission over an existing public fiber-optical network," in Opt. Fiber Commun. Conf. and Exhib. (2006).

13. L. Galdino et al., "An all-optical OCDMA encoder with simultaneous signal regeneration based on fiber four-wave mixing," Microwave Opt. Technol. Lett. 56(5), 1024-1028 (2014).

14. Q. Yu et al., "Secure $100 \mathrm{~Gb} / \mathrm{s} \mathrm{IMDD} \mathrm{transmission} \mathrm{over} 100 \mathrm{~km}$ SSMF enabled by quantum noise stream cipher and sparse RLS-Volterra equalizer," IEEE Access 8, 63585-63594 (2020).

15. D. Zhou et al., " $10 \mathrm{~Gb} / \mathrm{s}$ data transmissions using a widely tunable directly modulated InGaAlAs/InGaAsP DBR laser," IEEE Photonics Technol. Lett. 30(22), 1937-1940 (2018).

16. L. Han et al., "DBR laser with over 20-nm wavelength tuning range," IEEE Photonics Technol. Lett. 28(9), 943-946 (2016).

17. X. Xie et al., "Data transmission using a directly modulated widely tunable DBR laser with an integrated Ti thin film heater," IEEE Photonics J. 10(2), 1-6 (2018).

18. S. L. Woodward et al., "The side-mode-suppression ratio of a tunable DBR laser," IEEE Photonics Technol. Lett. 2(12), 854-856 (1990).

19. J. Buus, "Tunable laser sources for (D)WDM," Proc. SPIE 5280, 172-181 (2003).

20. J. Man et al., "An electrical-filtered optical heterodyne technique for tuning speed measurement of DBR lasers," Chin. Sci. Bull. 56(7), 704-708 (2011).

21. S. Zhu et al., "A simple photonic method to generate square and triangular microwave waveforms," Opt. Commun. 426, 654-657 (2018).

22. S. J. Zhang et al., "Electrical probing test for characterizing wideband optical transceiving devices with self-reference and on-chip capability," J. Lightwave Technol. 36(19), 4326-4336 (2018).

23. J. Sun et al., "The effect of bias and frequency on amplitude to phase conversion of photodiodes," IEEE Photonics J. 12(4), 1-10 (2020).

24. R. S. Tucker et al., "Propagation delays and transition times in pulse-modulated semiconductor lasers," Appl. Phys. Lett. 48(25), 1707-1709 (1986).

25. P. J. Rigole et al., "Fast wavelength switching in a widely tunable GCSR laser using a pulse pre-distortion technique," in Opt. Fiber Commun. Conf. and Exhib., pp. 231-232 (1997).

26. J. E. Simsarian et al., "Less than 5-ns wavelength switching with an SG-DBR laser," IEEE Photonics Technol. Lett. 18(4), 565-567 (2006).

Borui Xu received her BEng degree from the University of Electronic Science and Technology of China, Chengdu, China, in 2017. She is currently working toward her PhD in the Institute of Semiconductors, narrow linewidth tunable laser, Beijing, China. Her research interests include high speed direct-modulated semiconductor laser and narrow linewidth tunable laser.

Jiazheng Sun received his BSc degree from the University of Science and Technology Beijing, Beijing, China, in 2017. He is currently working toward her $\mathrm{PhD}$ at the Institute of Semiconductors, Chinese Academy of Science (CAS), Beijing, China. His research interests include high-speed photodiodes and narrow linewidth tunable laser.

Huashun Wen is currently an assistant professor with the Institute of Semiconductors, CAS, Beijing, China. His main study interests are microwave photonics, all-optical signal processing, and nonlinear optics. 
Xu et al.: Dual-pattern communication system using DBR laser in the intensity-modulation...

Ninghua Zhu is currently a professor with the Institute of Semiconductors, CAS, Beijing, China. He has authored or coauthored more than 200 journal papers, three books, and three book chapters. He was the recipient of the 2013 National Award for Technological Invention Second Prize and the 12th Guanghua Engineering Science and Technology Prize. He was a committee member of Information Department of the National Nature Science Foundation of China. 